\title{
¿Cómo percibe la banca cooperativa el impacto de la transformación dígital?
}

\author{
Yakira Fernández-Torres \\ Milagros Gutiérrez-Fernández \\ Ricardo Palomo-Zurdo
}

RESUMEN: El sector financiero en general, y el bancario en particular, se han caracterizado habitualmente por ser innovadores en la incorporación de la tecnología a sus procesos y servicios. Sin embargo, por primera vez en su historia, la tecnología está originando modos de desintermediación financiera y de capacidad de aportación de servicios que nacen en empresas tecnológicas y no en entidades financieras. La disrupción de las pequeñas empresas fintech; así como la innovación en servicios financieros ofrecidos por los gigantes tecnológicos, parecen amenazar el modelo de negocio tradicional de la banca. En este contexto, la banca cooperativa no es ajena a este fenómeno y vive su propia transformación digital, tanto por la demanda de sus socios y clientes, como por la necesidad de competir frente a esos nuevos actores financieros. Su tradicional arraigo territorial y el conocimiento más personalizado de sus socios y clientes deben hacerse ahora compatibles con los planteamientos de una nueva sociedad digital. Por ello, en este trabajo se ha analizado cuál es la percepción de las cooperativas de crédito españolas sobre la transformación digital, lo que ha arrojado interesantes resultados como: la reconocida necesidad de mejorar en la digitalización de sus procesos internos y externos; su percepción sobre la amenaza proveniente de las grandes empresas tecnológicas; las diferencias en lo que respecta a la digitalización de los socios y usuarios cuando cambia la dimensión de las entidades; así como su papel en la educación financierodigital de su base societaria y de usuarios.

PALABRAS CLAVE: cooperativas de crédito, banca cooperativa, digitalización, transformación digital, blockchain, experiencia de cliente.

CLAVES ECONLIT: G21, M15, 035, 039.

Cómo citar este artículo / How to cite this article: FERNÁNDEZ-TORRES, Y., GUTIÉRREZ-FERNÁNDEZ, M. \& PALOMO-ZURDO, R. (2019): “¿Cómo percibe la banca cooperativa el impacto de la transformación digital?", CIRIEC-España, Revista de Economía Pública, Social y Cooperativa, 95, 11-38. DOI: 10.7203/CIRIECE.95.12724.

Correspondencia: Yakira Fernández-Torres, yakiraft@unex.es, y Milagros Gutiérrez-Fernández, mgutierrezf@unex.es, profesoras doctoras, Departamento de Economía Financiera y Contabilidad, Facultad de Empresa, Finanzas y Turismo, Universidad de Extremadura, Avda. de la Universidad s/n, 10071, Cáceres, España; Ricardo Palomo-Zurdo, Catedrático de Universidad, Departamento de Economía Financiera y Contabilidad, Facultad de Ciencias Económicas y Empresariales, Universidad CEU San Pablo, C/ Julián Romea, $n^{\circ}$ 23, 28003, Madrid, España, palzur@ceu.es. 


\section{EXPANDED ABSTRACT}

\section{How do co-operative banks perceive the impact of digital transformation?}

The digital transformation is posing an unprecedented challenge for the financial sector. The classic model of financial intermediation is threatened. This is both a consequence of the development and the application of new technologies that are transforming society and that are empowering individuals with new digital tools.

Nowadays, banks are not only competing with each other. New actors have also emerged in the financial landscape, such as fintech (financial companies whose business model is technology-intensive) and techfin (technology companies offering financial services, especially large technology companies -or bigtech-). The extensive branch networks that are typical for traditional retail banking, such as in cooperative banks, are reducing their prominence in the face of the growing offer and digitized management of financial products and services.

The credit cooperatives have traditionally been characterized by a special link with their base of members and customers based on physical proximity. This has allowed the acquisition of great knowledge of the economic activity within the territory where the cooperative banks are established. Digital transformation and non-face-to-face financial services can significantly affect this traditional relational model based on personal contact with the users.

Consequently, faced with this paradigm shift and taking into account the particularities that define the cooperative banking model, there is an interest to analyse the perception of this sector on this important and accelerated technological change, both from the perspective of internal processes, as from the external perspective of its clients. At the same time, it is appropriate to inquire about whether this process is being perceived differently depending on the size of the cooperative bank, given the fact that the traditional model of cooperative banking has been based on a wide network of offices. This research would be, as far as it is known, the first to address such a task in the field of credit cooperatives, since the scarce studies found have a more general character about the banking sector as a whole.

In order to comply with the proposed objectives, information has been obtained through a questionnaire, consisting of 20 questions of a quantitative and qualitative nature. The questionnaire has been answered by a large number of Spanish credit cooperatives ( 41 out of 62 existing cooperative banks). With the obtained answers, a statistical-descriptive analysis has been completed, through the application of common statistics, such as: arithmetic mean, standard deviation, percentiles, maxi- 
mum and minimum. In turn, in line with the objectives set, statistics are presented including calculations for both, the sample set and for two sub-samples, in order to distinguish between entities of different dimensions.

Once the analysis has been concluded, some remarkable results have been obtained. On the one hand, the cooperative banks that have been included in this study have indicated the necessity to improve their digital transformation. It can also be observed that the digital gap is perceived in relation to the rest of the banking sector. Managers are aware of the significant impact of the digital transformation of the economy and society. Technology-based tools, such as big data, may have the greatest impact on the commercial banking activity. Within the next five years an intense competition from technology-based companies is expected. Furthermore, the technological culture of members and customers is insufficient.

On the other hand, it can also be observed that the dimension of the cooperative banks influences their perception of the digital transformation in the cooperative banking sector. In this sense, the cooperative banks that affirmed the existence of a wide margin of improvement in the operational scope have a smaller dimension and more seniority than the rest of the sample. Banks that have a higher number of total assets and a higher number of employees also perceive a greater margin of improvement when it comes to the internal management processes.

Additionally, the results indicate that the perceptions of digital transformation are conditioned by the size of the entity. Larger entities perceive a lower degree of assimilation of the digital transformation by the organization's employees, giving it greater importance to the impact of different technologies. Large entities perceive less intensity of competition from foreign banks and value the degree of technological and financial culture of members and customers more.

However, these results have limitations that have conditioned this research. These are basically founded on the fact that the analysis is based on the perceptions of the managers of the surveyed entities. This implies a degree of subjectivity conditioned by multiple non-controllable variables. With this general approach to different aspects related to digital transformation it has not been possible to contemplate other transcendental issues.

The study culminates with a series of strategic suggestions that can be considered by the sector. Credit cooperatives, usually with a smaller comparative dimension with respect to the large commercial banks and based on federative models, cooperation or systems of cooperative concentration give special value to territorial self-identification and proximity to the members. Technology can be a factor of distancing that can lead to a loss of personal contact with members or clients. Therefore, the cooperative bank must design compatibility strategies between the philosophy of its specific business model and the assimilation of digital transformation. Precisely, its closeness to the client can be the way to acquire a prominent role in the financial-digital education process that society will demand over the next few years. 
Additionally, based on the results obtained, it can be said that, from a strategic approach, credit cooperatives must value or advance in various lines of digital transformation of their activity, both in their internal and organizational processes and in their relations with clients. In the latter case, the reduction of processing times for operations, the use of apps (mobile applications) and access systems that are increasingly simple, agile and attractive, can be essential. Special attention should be given to anything that implies a more satisfied customer experience and simplification of procedures, with a wider and more personalized portfolio of financial products and services.

In this sense, the strategy should begin with evaluating the implementation of new technologies that already anticipate major changes. This is the case of blockchain in its internal processes or the use of vast amounts of data that is collected from the daily relationship with customers, with the aim of improving the personalization of the offer.

KEYWORDS: co-operative banks, co-operative banking, digitization, digital transformation, blockchain, customer experience. 


\section{Introducción'}

La notoria transformación digital que se está produciendo en todas las facetas de la sociedad tiene claras aplicaciones en el ámbito económico y, en particular, en el sector financiero.

La convergencia tecnológica, es decir, la concurrencia de diversas tecnologías, ha permitido crear nuevos dispositivos y nuevos servicios, hasta hace poco impensables. El desarrollo de la Internet de la información, que inició su popularización hace dos décadas, ha alumbrado ahora la Internet del valor (representado por la denominadas tecnologías distribuidas o DLT -Distributed Ledger Technologies-, como es el caso de blockchain) y se ha unido a ingeniosos sistemas colaborativos que están empoderando a los individuos con herramientas de gestión digital que amenazan muchos modelos clásicos de intermediación, como es el financiero (Lérida y Mora, 2016), pero también muchos modelos de gestión y de organización institucional; por lo que va a resultar especialmente trascendente durante los próximos años. Asimismo, hay otras tecnologías que resultan especialmente útiles en el sector financiero para sistemas de pronóstico y de análisis de comportamiento de la clientela mediante la explotación del llamado big data, que está generando ingente información que es fácilmente aprovechable por nuevos competidores de la banca: las grandes compañías tecnológicas, que son las grandes recolectoras de datos y que ha llevado a identificar a esos datos como el "petróleo del siglo XXI" (Chishti y Barberis, 2016).

Parte de la transformación digital de la sociedad es valorada como una revolución digital, no ajena a un clima de desconfianza de la población (BBVA Research, 2017) que se agudizó durante la mayor crisis financiera internacional conocida; razón por la cual, la tecnología se erige como un símbolo y un medio real de empoderamiento financiero, que ha originado el calificativo de democratización financiera, incluyendo el concepto de anarco-capitalismo, acuñado décadas atrás, y el más reciente criptoanarquismo (May, 1992).

El sector financiero y, en particular, las mayores entidades bancarias de todo el mundo, se han movilizado recientemente para afrontar, con diversos medios, el reto de la transformación digital, pero, especialmente, el reto de la competitividad de su modelo de intermediación financiera, cuyas barreras de entrada ya no parecen inexpugnables (Akkizidis y Stagars, 2016) como lo habían sido, hasta ahora, para las grandes compañías tecnológicas y para las más pequeñas -pero muy innovadoras- empresas del sector fintech. Si bien en la actualidad la mayor parte de los clientes bancarios se adhieren a los patrones de consumo tradicionales, en el futuro muchos de ellos serán expertos en el uso de tec-

1.- Los autores manifiestan que han contribuido de manera igualitaria en la realización del artículo, realizado con la colaboración de la Cátedra "José Barea" de Hacienda Pública y Economía Social del Instituto de Estudios Fiscales del Ministerio de Hacienda y AAPP, de la Fundación para la Innovación Financiera y la Economía Digital (FIFED) y, particularmente, de la Unión Nacional de Cooperativas de Crédito (UNACC) y del Grupo Cajamar, sin cuyo apoyo habría resultado muy difícil la elevada tasa de respuesta obtenida en la encuesta realizada. 
nologías como resultado del cambio demográfico (Dapp, 2015), por lo que debe tenerse en cuenta que un elemento clave es la búsqueda de la mejora de la experiencia de cliente tanto desde el momento de su adhesión inicial, como durante la relación cotidiana con sus entidades. Por tanto, la banca está en obligada transición desde un modelo de banca de productos a un modelo de banca de clientes, añadiendo la experiencia de servicio por parte de sus usuarios, que se considera ya fundamental a efectos reputacionales y competitivos. Debe tenerse en cuenta que el descontento -o el agrado- de un usuario con respecto al servicio recibido trascendía, años atrás, sólo a un círculo cercano de amistades o familiares. En cambio, actualmente, las redes sociales pueden potenciar exponencialmente esta transmisión de experiencias, por lo que la experiencia y satisfacción del cliente ocupan una posición de primer orden.

En paralelo, las amplias redes de oficinas características de la banca minorista tradicional van reduciendo su protagonismo frente a la creciente digitalización de la población. Así, los datos avalan que el avance del sector aún es insuficiente respecto a la evolución de los clientes bancarios, pues muchos bancos no están preparados. Concretamente, en el caso de Europa los bancos minoristas han digitalizado tan sólo entre el 20 y el $40 \%$ de sus procesos y en el $90 \%$ de estas entidades la inversión digital es inferior al 0,5\% de su gasto total (Olanrewaju, 2014).

Sin embargo, el impacto de la crisis financiera iniciada en 2007 inició un proceso de sustancial reducción del número de oficinas y de empleados en la mayoría de las entidades bancarias de tipo comercial o minorista; y es, precisamente en este segmento de la actividad bancaria, en el que siempre se ha desarrollado la función financiera de las cooperativas de crédito, con una especial vinculación con su base de socios y clientes fundamentada en la proximidad física. Esto ha sido lo que ha proporcionado a la banca cooperativa un gran conocimiento de la actividad económica del territorio donde se encuentra implantada, por lo que la transformación digital y los servicios financieros no presenciales pueden afectar a este modelo relacional tradicional basado en el contacto personal con el usuario.

Con todo, cabe apreciar que la transformación tecnológica de la sociedad, intensiva en modelos colaborativos y de compartición, así como en sistemas directos de interrelación a través de plataformas digitales, comparte algunos principios con la concepción cooperativa; hasta el punto de que se ha acuñado el concepto de "economía de plataforma".

En este sentido, procede reseñar que la fórmula cooperativa se visiona ya como un sistema societario que puede adaptarse bien a los modelos distribuidos que, de la mano del blockchain y de otras tecnologías similares, comenzarán a extenderse en los próximos años. Así, es posible que la fórmula cooperativa pueda aportar un sistema de gobernanza para la tecnología que más puede llegar a cambiar los paradigmas económicos; pero, mientras tanto, y sin entrar en cuestiones societarias y de buen gobierno en la fórmula cooperativa (Chaves y Soler, 2014), la prioridad estratégica es ver cómo las cooperativas de crédito se adaptan a la transformación digital y la ejecutan de forma efectiva, competitiva y sin desnaturalizar su identidad y sus funciones. 
En este contexto de cambio de paradigma de la economía y de la sociedad y de replanteamiento del modelo de intermediación financiera, con la inquietud que representa el surgimiento de nuevos competidores en un sector actualmente tan regulado, como es el financiero, procede analizar cuál es la percepción de la banca cooperativa sobre este cambio, tanto desde la perspectiva interna de sus procesos, como desde la perspectiva externa de sus usuarios. A la vez, resulta conveniente indagar sobre si dicho proceso es percibido de manera diferente según la dimensión de la entidad, dado que el modelo tradicional estructural del sector bancario y, concretamente, el cooperativo, se ha sustentado en un amplio entramado de oficinas y empleados, todo lo cual implica la necesidad de una profunda transformación para adaptarse al nuevo contexto que se está configurando.

Por ello, esta investigación aborda ambas perspectivas y presenta los resultados de un estudio sobre el sector de la banca cooperativa española, basado en un cuestionario en el que han participado una gran mayoría de las cooperativas de crédito (41 de las 62 existentes). Con las respuestas obtenidas se ha realizado un análisis de tipo estadístico descriptivo, del cual destaca la importancia que el sector otorga a la transformación digital, la necesidad que reconocen de avanzar en este sentido, la intensa amenaza que estiman en el futuro proveniente de la competencia de empresas de base tecnológica, el impacto significativo del big data en la actividad, la aún insuficiente cultura tecnológica de socios y clientes y las diferencias en percepciones existentes al comparar entre dos conjuntos de entidades, obtenidos al dividir la muestra entre las de mayor y menor tamaño. Hasta donde se conoce, los estudios sobre transformación digital en el sector cooperativo español son inexistentes; más bien, lo que se ha hecho abarca al sector bancario en general (Jaubert, et al. 2014; Herráiz et al., 2015), por lo que este sería el primero en abordar esta cuestión en el ámbito de las cooperativas de crédito.

La estructura de este trabajo consta de esta introducción, seguida del marco teórico que atiende a la perspectiva de las cooperativas de crédito y los desafíos que supone la transformación digital. En tercer lugar, se analiza la muestra y se presenta la metodología aplicada; para continuar con el cuarto apartado centrado en los resultados, finalizando el trabajo con las conclusiones.

\section{El sector de las cooperativas de crédito: retos ante la transformación digital}

El sector español de las cooperativas de crédito ha sido el menos afectado, en términos financieros, por la crisis económica de los años 2008 a 2014, que hizo desaparecer a las cajas de ahorros y que obligó a hacer importantes ajustes en los bancos. En la figura 1 puede apreciarse cuál es el actual mapa del sistema bancario español, recogiendo su dimensión sectorial comparativa y su estructura representativa. 
Figura 1. Las cooperativas de crédito en el mapa del sistema bancario español

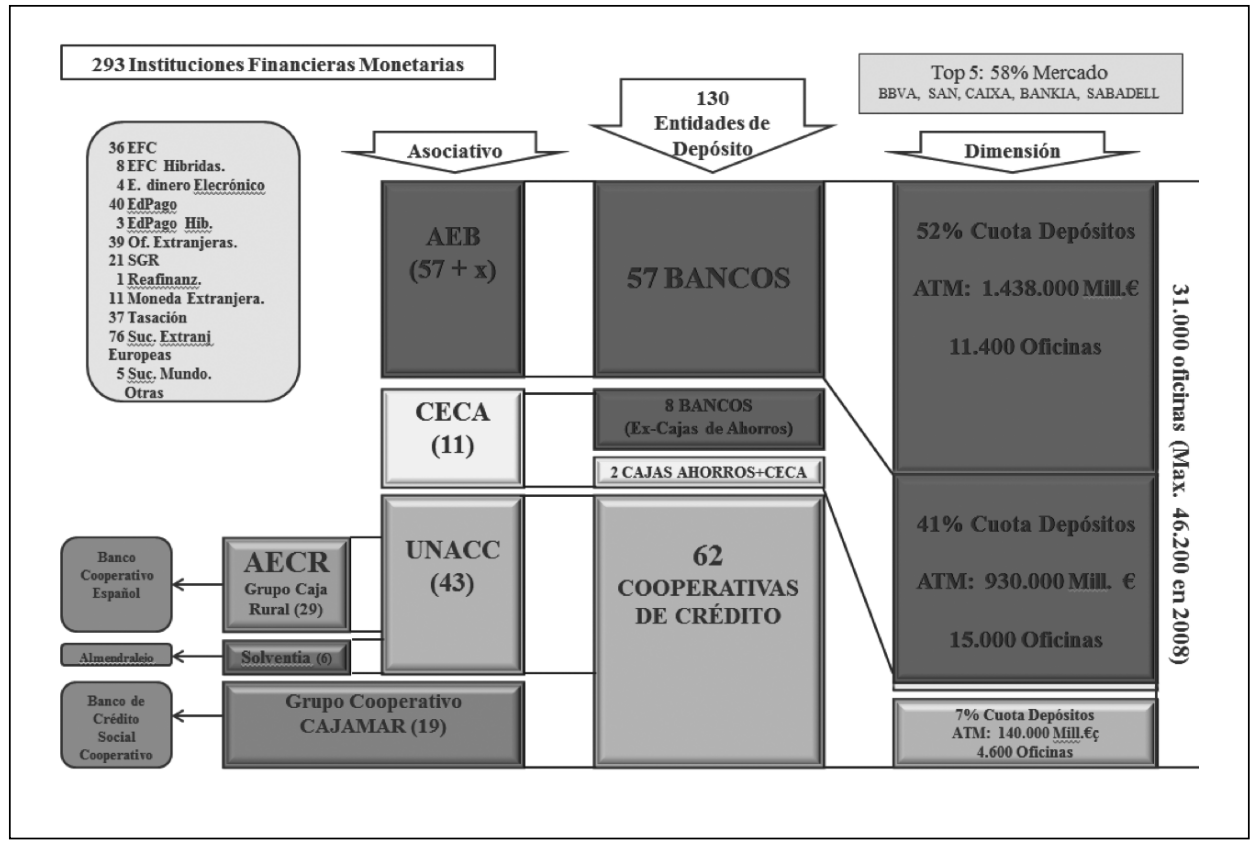

FUENTE: Palomo (2017).

Al contrario que los bancos y las extintas o transformadas cajas de ahorros, la banca cooperativa no ha experimentado una variación significativa de oficinas ni reducción de empleo como consecuencia de la crisis financiera; en parte, debido a que ha mantenido su posición de fuerte presencia territorial (Gutiérrez et al., 2012) y, también, porque ha cubierto áreas territoriales en las que las cajas de ahorros se replegaron durante la crisis; a pesar de que el sector bancario ha experimentado, globalmente, la mayor y más intensa reducción del número de oficinas y de empleados de su historia (King, 2014).

Sin embargo, la banca cooperativa española sí que ha experimentado una profunda reestructuración, principalmente mediante la concentración de muchas entidades del sector (Palomo y Sanchis, 2010) y la constitución de diversos grupos cooperativos, con aplicación de la fórmula de los Sistemas Institucionales de Protección (SIP), donde los integrantes ponen en común -en diverso grado- su solvencia y los resultados de la actividad (Gutiérrez y Palomo, 2012; Fajardo y Soler, 2016). 
Actualmente, la banca cooperativa en España cuenta con 62 cooperativas de crédito que superan los 2,7 millones de socios y los 11 millones de clientes; con un activo de 140.000 millones de euros y una cuota de mercado del $6,75 \%$ de los depósitos y del 6,1\% de los créditos. Cuenta con casi 20.000 empleados y 4.600 oficinas? 2 .

Un aspecto que dota de especial fortaleza al modelo competitivo de las cooperativas de crédito es su extraordinaria adaptación a sus socios y clientes y el establecimiento de un estrecho vínculo con ellos (EACB, 2014; Oliver Wyman, 2014), que deberían mantener pese al posible distanciamiento personal que la tecnología puede provocar en la operativa diaria, como ocurre con los sistemas de banca a distancia que conviven con la atención personalizada en la oficina. La experiencia de cliente se revela ahora como un aspecto crucial ante la simplicidad de muchos procesos de intermediación financiera que ofrecen las compañías fintech ${ }^{3}$ y las grandes tecnológicas; por lo que se precisa una personalización del servicio a pesar de que en ello se involucren tecnologías como la inteligencia artificial (para la atención al cliente) 0 el asesoramiento robotizado. Así, no deja de ser controvertido el hecho de que sea la tecnología lo que ayude a "personalizar" la relación con los usuarios.

En este sentido, si bien, es precisa la innovación en la prestación de servicios (Chesbrough, 2010) se debe, a la vez, mantener una posición competitiva en un entorno en el que han entrado nuevos actores (Carbó y Rodríguez-Fernández, 2014, 2016, y 2017; Maudos, 2017) como las referidas fintech y, próximamente, las grandes tecnológicas.

La reforma del sector financiero tiene conexión con el crecimiento de la banca digital (Shaikh et al., 2017; Bansal y Jain, 2018); y se trata de una transformación sin precedentes, por la velocidad del cambio y por incorporar el concepto de tener que construir un nuevo sistema financiero o su propia deconstrucción (Evans y Wurster, 1999), que funciona como un proceso creativo o de innovación abierta (Chesbrough, 2003 y 2010).

Las cooperativas de crédito, al igual que el conjunto del sector bancario, necesitan adaptar su modelo organizativo y rediseñar una parte sustancial de su modelo tradicional de negocio (Morris, 2006; Giesen et al., 2010) facilitando una cultura de innovación abierta que esté alineada con sus usuarios para que resulte viable (Zott y Amit, 2007), además de generar un nuevo modelo de confianza tras el descrédito del sector financiero durante la crisis económica.

En este sentido, cabe indicar que la nueva economía digital, de base colaborativa, se atribuye sistemas de confianza entre las partes, aunque no haya contacto directo ni conocimiento entre ellos. La

2.- Datos tomados agregando el conjunto del sector en España, partiendo de las entidades vinculadas al Grupo Cajamar (19) y las asociadas a la Unión Nacional de Cooperativas de Crédito UNACC (43). Además, hay dos entidades en liquidación (Caja de Crédito de Alcoy y Caja Rural de la Carlota).

3.- Para mayor información sobre el sector fintech y su impacto en el sistema financiero y bancario véase Jakšic y Marinc (2018), Blomstrom (2018) o Gomber et al. (2018), entre otros. 
valoración que los clientes hacen permanentemente de los servicios recibidos tiene un gran impacto en la opinión global y en las redes sociales. De hecho, esos sistemas de confianza colaborativos son los que fundamentan muchos modelos de las llamadas economías de plataforma; por lo que también las entidades bancarias se ven obligadas a reforzar el concepto de confianza con sus clientes (Mukherjee y Nath, 2003).

Otro factor a tener en cuenta es el relativo a los ajustes en la plantilla y de las redes de oficinas de la banca española como consecuencia de la crisis. También el que concierne al cambio tecnológico y la reducción de costes que aporta la banca a distancia, que ha creado situaciones de "exclusión financiera" ya que, en 2017 , cerca del $48 \%$ de los municipios españoles y 1,13 millones de ciudadanos no tenían acceso a una oficina bancaria (Maudos, 2017), lo que puede suponer profundizar en la "brecha digital" de amplias capas de la sociedad española.

Desde otro punto de vista, el conjunto del sector financiero se ha visto afectado por las consecuencias de las malas prácticas sobre la imagen de la banca (Sanchis, 2016), lo que también está motivando mayor interés del público por nuevas formas de intermediación financiera. Los usuarios demandan una mejor experiencia de cliente (Mbama y Ezepue, 2018), por lo que es preciso construir un modelo de banca viable en el nuevo contexto digital, rentable en el competitivo entorno financiero y, también, "amable" con los clientes (Massanell, 2016).

Por otra parte, hay que tener en cuenta el impacto que va a tener el denominado "open banking" como consecuencia de la aplicación de la directiva europea conocida como PSD2 4 o segunda directiva de servicios de pago, que obliga, desde comienzos de 2018, a que los bancos cuenten con procedimientos tecnológicos (conocidos como $\mathrm{APIs}^{5}$ ) que habiliten el acceso a los datos de los clientes por parte de otras entidades del sector fintech, siempre que éstas sean autorizadas por sus clientes, lo que ha permitido acuñar el término de Api-economía (Zachariadis y Ozcan, 2017).

En suma, son muchos y muy rápidos los cambios que acontecen en el sector financiero y la banca cooperativa no es ajena a ellos, destacando la doble amenaza del sector fintech y de los gigantes tecnológicos (Gomber et al., 2018). Por tanto, para sobrevivir en este nuevo contexto competitivo, el sector bancario deberá deconstruir su cadena de valor y reestablecerla integrando la cultura de la transformación digital (Herráiz et al., 2015). A esto cabe añadir el futuro impacto de la progresiva introducción de criptomonedas, así como la progresiva desaparición del dinero físico en forma de monedas y billetes 6 (Skinne, 2016; HM Treasury, 2015, Taylor, 2014 y The Economist Leaders, 2015).

Adicionalmente, la más reciente de esas tecnologías, que promete incidir fuertemente sobre la actividad bancaria tradicional, es la citada blockchain, que surge como una solución a los graves

4.- Second Payments Services Directive (PSD2).

5.- Application Programming Interfaces (API).

6.- Por ejemplo, en Dinamarca, a partir del año 2017, cualquier empresa o negocio podrá negarse a aceptar pagos en dinero efectivo. 
problemas de la vulnerabilidad de la Internet de la información o tradicional, además de ser un medio de transferir órdenes y transacciones de modo indescifrable. De hecho, su primera aplicación real fue en el ámbito financiero, con criptomonedas, criptoactivos o criptodivisas?

Otro aspecto derivado a tener en cuenta es que algunos grandes bancos han comenzado a adquirir empresas fintech para incorporar sus nuevos modelos de relación con los clientes a sus tradicionales estructuras, tratando de aprehender y de aprender de estos nuevos actores (Busby, 2017) en un intento de ofrecer mejores servicios a sus clientes, e incluso, de promover su cultura financiero-digital (Rahman, 2017).

Por primera vez en la historia, el desarrollo de la tecnología permite la participación global de usuarios privados e institucionales de todo el mundo sobre un mismo tablero de juego.

\section{Obtención de la muestra y metodología de análisis}

La muestra de este estudio se ha conformado a partir de las respuestas obtenidas mediante un cuestionario on line gestionado de forma anónima, que fue elaborado bajo el soporte de la plataforma Eval\&Go y remitido a las 62 cooperativas de crédito españolas con el apoyo de la Unión Nacional de Cooperativas de Crédito (UNACC) y el Grupo Cajamar.

Dicho cuestionario, compuesto de 20 preguntas de tipo cualitativo y cuantitativo, fue accesible durante el período comprendido entre el 20 de marzo y el 25 de abril de 2018. Del total de entidades a las que se remitió la solicitud, se recibió respuesta de 41 de ellas, es decir, las dos terceras partes de la población de interés. En el anexo 1 se recogen estos datos conformando la ficha técnica de la muestra.

Dado que este trabajo representa la continuación de una investigación inicial, se han tomado para el análisis que se desarrolla seguidamente sólo las preguntas cuyas respuestas hicieron posible la obtención de estadísticos descriptivos, por lo que la investigación se fundamentará en 12 de las 20 preguntas del cuestionario y que se concretan en la tabla 1.

7.- La primera aplicación real de la tecnología blockchain se realizó con la conocida criptomoneda Bitcoin, originada con la publicación, en el año 2008, del ya famoso documento firmado por Satoshi Nakamoto (pseudónimo de una identidad real aún desconocida) titulado "Bitcoin: A Peer-to-Peer Electronic Cash System" que puede verse en: https://bitcoin.org/bitcoin.pdf. 


\section{Tabla 1. Cuestionario}

\begin{tabular}{|l|l|}
\hline Preguntas & Contenido \\
\hline $1-4$ & Activo total, número de empleados y oficinas y antigüedad. \\
\hline $5-11$ & $\begin{array}{l}\text { Margen de mejora de la transformación digital, su grado de asimilación por los diferentes com- } \\
\text { ponentes y percepción de socios y clientes sobre la adaptación tecnológica. } \\
\text { Existencia de brecha digital en comparativa al resto del sector bancario. } \\
\text { Percepción del impacto económico de la transformación digital. } \\
\text { Tipo de tecnologías que más afectan a la actividad y con qué importancia. } \\
\text { Intensidad de la competencia prevista de diferentes actores. }\end{array}$ \\
\hline 12 & Cultura financiera y tecnológica de socios y clientes. \\
\hline
\end{tabular}

FUENTE: Elaboración propia a partir del cuestionario.

Como se puede apreciar en dicha tabla, las primeras cuatro preguntas fueron destinadas a conocer las características fundamentales de las entidades analizadas: dimensión (a través del activo total, número de empleados y de oficinas) y antigüedad (en el caso de fusiones, se tomó como año inicial, el año de la fusión o proceso de concentración, como por ejemplo, la constitución de un SIP o grupo cooperativo). En el caso de las restantes preguntas, se indaga sobre la transformación digital desde diferentes perspectivas, como son: la de sus grupos de interés, la comparación respecto al resto del sector bancario, la importancia para la actividad económica y los tipos de tecnologías que más afectan a la actividad. De esta forma, ambos conjuntos de preguntas permitirán, no solamente la realización una descripción de las percepciones sobre el proceso de transformación digital en el sector cooperativo de crédito, sino también conocer las condiciones de las entidades según los diferentes grados de transformación digital y si éstos están explicados por la dimensión de la organización, en línea con lo pretendido en este trabajo. Para la consecución de estas metas, se llevará a cabo un análisis estadístico descriptivo mediante el uso de estadísticos comunes, como son: media aritmética, desviación típica, percentiles, máximo y mínimo. 


\section{Resultados del análisis y aplicación para la orientación estratégica de la banca cooperativa}

Este apartado se inicia con una breve exposición de algunas características distintivas de la muestra de entidades analizadas, basada en la información de la tabla 2, que aglutina estadísticos descriptivos relativos al tamaño y la antigüedad de dichas organizaciones. La antigüedad media aproximada de las cooperativas de crédito analizadas es de 46 años, si bien el $75 \%$ no alcanza los 60 años. El $75 \%$ de la muestra posee un activo total inferior a 2.798 millones de euros, menos de 400 empleados y de 112 oficinas. El promedio de activo total, empleados y oficinas es, respectivamente, de 3.487 millones de euros, 447 empleados y 100 oficinas; existiendo una gran heterogeneidad en términos de dimensión, como reflejan las desviaciones típicas de estas tres variables y los diferenciales entre sus respectivos valores máximo y mínimo.

\section{Tabla 2. Respuestas a preguntas 1-4: características de la muestra}

\begin{tabular}{|l|c|c|c|c|c|c|c|c|}
\hline Var & Obs & Media & $\begin{array}{c}\text { Desv } \\
\text { Típica }\end{array}$ & Máx & Mín & Perc25 & Perc50 & Perc75 \\
\hline Activo Total (mill €) & 41 & 3.487 & 8.038 & 44,604 & 20 & 114 & 519,027 & 2.798 \\
Empleados & 41 & 447,37 & 863,60 & 4.418 & 4 & 23 & 50 & 400 \\
№ Oficinas & 41 & 99,54 & 184,71 & 987 & 1 & 4 & 13 & 112 \\
Antigüedad (años) & 38 & 45,76 & 28,90 & 101 & 5 & 15,50 & 50,50 & 59,25 \\
\hline
\end{tabular}

FUENTE: Elaboración propia a partir de los resultados del cuestionario.

En lo referente al análisis sobre la transformación digital, es necesario indicar que, en concordancia con los objetivos pretendidos, se ha dividido en tres partes. En primer lugar, se muestran los estadísticos descriptivos de cada una de las respuestas a las preguntas formuladas, con el fin de mostrar una visión general de la valoración que éstas han recibido por parte de los encuestados, teniendo en cuenta que 0 significa nada y 5 mucho (véase tabla 3). La tabla 4 refleja la media aritmética de las características de las entidades antes mencionadas en función del diferente grado de valoración sobre la transformación digital, a partir de una selección de preguntas. De esta forma, se pueden analizar las diferencias entre entidades según distintos grados de percepción sobre transformación digital. En tercer lugar, con la tabla 5 se presenta un análisis de dichas percepciones según la dimensión, permitiendo así conocer si el tamaño de la entidad condiciona su postura respecto al proceso de transformación digital. 
Comenzando con el análisis de los estadísticos descriptivos mostrados para cada pregunta en la tabla 3 , cabe destacar los siguientes resultados.

En primer lugar, las respuestas a la pregunta 5 indican la consideración de que existe un amplio margen de mejora en términos de transformación digital, tanto en la operativa como en los procesos de gestión interna. Ello se constata en las puntuaciones medias, que son 3,53 y 3,63, respectivamente (sobre la escala de 0 a 5), así como en el valor mínimo asignado (2), por lo que en ningún caso se ha respondido con 001 , y en que el $75 \%$ de las entidades asignó una valoración de 30 más.

En segundo lugar, cuando se observan las respuestas relativas al grado de asimilación de la transformación digital entre los diferentes componentes de la entidad (pregunta 6), se aprecia que sobresalen dos conjuntos, según las puntuaciones recibidas: los que tienen una valoración media superior a 3 (Dirección General y equipo directivo y empleados) y los que no alcanzan dicho valor (Consejo de Administración y Presidencia y socios y clientes). Además, en el caso de los primeros, el $75 \%$ de las entidades le asignó una numeración de 3 o más, mientras que para los segundos la puntuación mínima fue de 20 más. A su vez, sólo en la valoración relativa al Consejo de Administración y Presidencia se asignó la numeración 0 , es decir, la que indica que el grado de asimilación es nulo. Por tanto, los datos reflejan una diferencia entre los componentes con vinculación directa a la entidad y los que no la tienen, tal que se percibe de los primeros una mayor asimilación de la transformación digital que de los segundos.

Seguidamente, las preguntas 7,8 y 9 hacen referencia a la valoración sobre cómo perciben los socios y clientes la adaptación tecnológica de la entidad a sus necesidades, a si existe brecha digital en el sector de cooperativas de crédito en comparativa al resto de la banca y al impacto que está teniendo la transformación digital de la economía, respectivamente. A priori, llama la atención que las puntuaciones más altas fueron otorgadas en la pregunta 9 , es decir, el $75 \%$ de las entidades asignó un 4 o más de valoración, lo que derivó en una puntuación media de 4,05, indicando así la importancia que otorgan al efecto de la transformación digital de la actividad económica. En el caso contrario está la pregunta 8 con los valores más bajos (promedio: 2,63), reflejando que, a pesar de reconocer la existencia de brecha respecto al resto del sector bancario, ésta no se percibe como amplia. Por último, la puntuación media de 3,07 de la pregunta 7 hace concluir que las entidades encuestadas consideran que los clientes perciben una adecuada adaptación tecnológica a sus necesidades de productos y servicios.

Por otra parte, resulta de especial interés conocer las tecnologías que más afectan a la actividad de las entidades estudiadas (pregunta 10). En este sentido, el big data es el que recibe mayor reconocimiento con diferencia, con una puntuación media de 4,07 y el valor mínimo más alto (2). A éste le sigue en valoración el cloudcomputing y el blockchain, con valores medios de 3,34 y 3,32, respectivamente. Al observar las tecnologías menos valoradas, en el extremo se encuentra la realidad aumentada y virtual, con una numeración media de 2,19. 


\section{Tabla 3. Estadísticos descriptivos obtenidos a partir de las respuestas a cada pregunta}

\begin{tabular}{|c|c|c|c|c|c|c|c|}
\hline Pregunta & Media & $\begin{array}{l}\text { Desv. } \\
\text { Estándar }\end{array}$ & Mín & Máx & Perc25 & Perc50 & Perc75 \\
\hline \multicolumn{8}{|l|}{ 5- Margen de mejora (0 -nada- a 5 -mucho-): } \\
\hline Operativa con productos, servicios y clientes & 3,53 & 0,71 & 2 & 5 & 3 & 4 & 4 \\
\hline Procesos de gestión interna & 3,63 & 0,82 & 2 & 5 & 3 & 4 & 4 \\
\hline \multicolumn{8}{|c|}{ 6- Grado de asimilación de la transformación digital (0 -nada- a 5 -mucho-): } \\
\hline Consejo de Administración y Presidencia & 2,90 & 1,09 & 0 & 5 & 2 & 3 & 4 \\
\hline Dirección General y equipo directivo & 3,63 & 0,96 & 1 & 5 & 3 & 4 & 4 \\
\hline Resto de empleados & 3,41 & 0,80 & 2 & 5 & 3 & 3 & 4 \\
\hline Socios y clientes & 2,80 & 0,81 & 1 & 4 & 2 & 3 & 3 \\
\hline \multicolumn{8}{|c|}{ 7- Percepción de socios y clientes de adaptación tecnológica a sus necesidades ( 0 -nada- a 5 -mucho-): } \\
\hline & 3,07 & 0,81 & 1 & 5 & 3 & 3 & 4 \\
\hline \multicolumn{8}{|c|}{ 8- Existencia de brecha digital con respecto al resto del sector bancario ( 0 -nada- a 5 -mucho-): } \\
\hline & 2,63 & 1,17 & 0 & 5 & 2 & 3 & 4 \\
\hline \multicolumn{8}{|c|}{ 9- Percepción del impacto de la transformación digital de la economía (0 -nada- a 5 -mucho-): } \\
\hline & 4,05 & 0,81 & 2 & 5 & 4 & 4 & 5 \\
\hline \multicolumn{8}{|c|}{ 10- Tecnologías que afectan más a la actividad y con qué grado de importancia ( 0 -nada- a 5 -mucho-): } \\
\hline Big Data & 4,07 & 0,98 & 2 & 5 & 4 & 4 & 5 \\
\hline Blockchain & 3,32 & 1,14 & 1 & 5 & 2,5 & 3 & 4 \\
\hline Criptomonedas & 2,26 & 1,26 & 0 & 5 & 1 & 2 & 3 \\
\hline Roboadvisor & 3,19 & 1,28 & 0 & 5 & 2 & 4 & 4 \\
\hline Inteligencia Artificial & 3,31 & 1,12 & 0 & 5 & 3 & 3 & 4 \\
\hline Internet de las Cosas & 2,29 & 1,20 & 0 & 4 & 1 & 2 & 3 \\
\hline Cloudcomputing & 3,34 & 1,23 & 1 & 5 & 3 & 3 & 4 \\
\hline Realidad aumentada y virtual & 2,19 & 1,07 & 0 & 4 & 1 & 2 & 3 \\
\hline Asistentes virtuales domésticos & 2,41 & 1,16 & 0 & 4 & 2 & 2 & 3 \\
\hline \multicolumn{8}{|c|}{ 11- Intensidad de la competencia en los próximos 5 años ( 0 -nada- a 5 -mucho-): } \\
\hline Desde otras entidades financieras similares & 3,09 & 1,20 & 1 & 5 & 2 & 3 & 4 \\
\hline De otras entidades bancarias de mayor dimensión & 4,34 & 0,82 & 2 & 5 & 4 & 5 & 5 \\
\hline De la banca extranjera & 2,60 & 1,35 & 0 & 5 & 2 & 2 & 4 \\
\hline De los bancos "on line" & 3,85 & 0,98 & 2 & 5 & 3 & 4 & 5 \\
\hline Del sector fintech & 3,63 & 1,17 & 0 & 5 & 3 & 4 & 5 \\
\hline De las grandes empresas tecnológicas & 3,58 & 1,16 & 1 & 5 & 3 & 4 & 4 \\
\hline \multicolumn{8}{|c|}{ 12- Cultura financiera y tecnológica de socios y clientes (0 -nada- a 5 -mucho-): } \\
\hline Grado general de cultura financiera & 2,78 & 0,88 & 1 & 5 & 2 & 3 & 3 \\
\hline Grado general de cultura tecnológica & 2,51 & 0,71 & 1 & 4 & 2 & 2 & 3 \\
\hline $\begin{array}{l}\text { ¿Puede contribuir su entidad a aumentar la cultura } \\
\text { financiera de socios y clientes? }\end{array}$ & 3,80 & 0,78 & 2 & 5 & 3 & 4 & 4 \\
\hline $\begin{array}{l}\text { ¿Puede contribuir su entidad a aumentar la cultura } \\
\text { tecnológica de socios y clientes? }\end{array}$ & 3,63 & 0,91 & 2 & 5 & 3 & 4 & 4 \\
\hline
\end{tabular}

FUENTE: Elaboración propia a partir de los resultados del cuestionario. 
Respecto a las preguntas restantes, la 11 refleja información sobre la intensidad de la competencia esperada desde diferentes ámbitos en los próximos cinco años, mientras que la 12 se centra en el grado de cultura financiera y tecnológica de socios y clientes. En cuanto a la primera, los resultados avalan la importancia otorgada a la competencia proveniente de empresas de base tecnológica. Específicamente, si bien se espera una competencia más intensa de las grandes entidades bancarias (puntuación media: 4,34), los siguientes competidores que más preocupan son los bancos on line (puntuación media: 3,85 ), el sector fintech (puntuación media: 3,63) y las grandes empresas tecnológicas $(3,58)$. En lo referente a la segunda, los datos reflejan que las entidades participantes perciben, en general, una escasa cultura financiera y tecnológica de socios y clientes, siendo más acentuado en el ámbito de la tecnología. No obstante, se muestran optimistas en el papel de la entidad financiera en la contribución a la mejora de las mismas.

Habiendo reflexionado sobre las cuestiones que distinguen a los resultados obtenidos, a continuación, el debate se centrará en la relación entre las características de las entidades y las percepciones de éstas sobre el proceso de transformación digital, teniendo en cuenta la puntuación que las mismas asignan a diversos aspectos de dicho proceso (se realiza una selección, dada la extensión). Para ello, se comenzará con la tabla 4, que recoge la media aritmética del activo total, número de empleados, número de oficinas y antigüedad, diferenciando dos posibles valores: si la puntuación alcanza al menos el 3 o lo contrario, considerándose así una valoración alta en el primer caso y baja en el segundo. Es necesario aclarar que se ha tomado dicho valor como referencia para distinguir entre valores altos y bajos porque, al tratarse de una puntuación que abarca seis valores posibles ( 0 a 5), el 3 representa el primero de los tres más altos.

Así, al observar la tabla 4, llama la atención que existen, en general, diferencias notables en términos de dimensión y antigüedad entre las empresas analizadas, según si éstas otorgan una valoración baja ( $<3) 0$ alta $(>=3)$ a las cuestiones preguntadas sobre transformación digital; si bien hay que tener en cuenta que, en prácticamente todos los casos, son muchas más las empresas que asignan una puntuación alta.

Comenzando el análisis con las percepciones sobre el margen de mejora de la entidad en relación con la transformación digital (criterios 1 y 2), las entidades que asignaron una puntuación elevada en el ámbito de la operativa con productos, servicios y clientes tienen, en promedio, un menor número de empleados (356 frente a 2.230) y oficinas (79 frente a 496) y más antigüedad (47 frente a 24 años) que las entidades que otorgaron una valoración baja, tratándose de diferenciales acusados. Es decir, las cooperativas más ligeras en estructura y más experimentadas estiman una mayor necesidad de mejora en la evolución de la transformación digital que afecta a la operativa. Sin embargo, en lo referente a la mejora en los procesos de gestión interna, las entidades que afirmaron la existencia de un amplio margen de mejora tienen, en promedio, mayor activo total y número de empleados.

Seguidamente, pueden observarse los datos promedios según el grado de asimilación de la transformación digital por componentes de la entidad (criterios 3 a 6). Así, las cooperativas de crédito que 


\section{Tabla 4. Media aritmética de características de las entidades según diferentes criterios sobre transformación digital, teniendo en cuenta que 0 significa nada y 5 mucho}

\begin{tabular}{|c|c|c|c|c|}
\hline Puntuación Criterio & Activo Total (€) & Empleados & $\mathrm{N}^{\circ}$ Oficinas & Antigüedad (años) \\
\hline \multicolumn{5}{|c|}{ Criterio 1: Margen de mejora en la operativa con productos, servicios y clientes } \\
\hline >=3 (39 empresas) & $2.52 \mathrm{e}+09$ & 355,9487 & 79,17949 & 46,94444 \\
\hline$<3$ (2 empresas) & $2.24 \mathrm{e}+10$ & 2230 & 496,5 & 24,5 \\
\hline \multicolumn{5}{|c|}{ Criterio 2: Margen de mejora en los procesos de gestión interna } \\
\hline$>=3$ (38 empresas) & $3.56 \mathrm{e}+09$ & 458,21 & 97,36 & 44,82 \\
\hline$<3$ (2 empresas) & $2.54 e+09$ & 310 & 127 & 56,66 \\
\hline \multicolumn{5}{|c|}{ Criterio 3: Asimilación de la transformación digital del Consejo de Administración y Presidencia } \\
\hline$>=3$ (29 empresas) & $4.54 \mathrm{e}+09$ & 574,44 & 124,82 & 46,80 \\
\hline$<3$ (12 empresas) & $9.40 \mathrm{e}+08$ & 140,25 & 38,41 & 43,50 \\
\hline \multicolumn{5}{|c|}{ Criterio 4: Asimilación de la transformación digital de la Dirección General y equipo directivo } \\
\hline >=3(37 empresas) & $3.61 e+09$ & 461,54 & 102,27 & 46 \\
\hline$<3$ (4 empresas) & $2.32 \mathrm{e}+09$ & 316,25 & 74,25 & 43,75 \\
\hline \multicolumn{5}{|c|}{ Criterio 5: Asimilación de la transformación digital del resto de empleados } \\
\hline >=3 (37 empresas) & $3.54 \mathrm{e}+09$ & 457,18 & 100,27 & 44,05 \\
\hline$<3$ (4 empresas) & $3.03 e+09$ & 356,50 & 92,75 & 60,25 \\
\hline \multicolumn{5}{|c|}{ Criterio 6: Asimilación de la transformación digital de socios y clientes } \\
\hline >=3 (27 empresas) & $2.52 \mathrm{e}+09$ & 375,40 & 68,59 & 50,96 \\
\hline$<3$ (14 empresas) & $5.35 e+09$ & 586,14 & 159,21 & 34,50 \\
\hline \multicolumn{5}{|c|}{ Criterio 7: Percepción de socios y clientes de adaptación tecnológica a sus necesidades } \\
\hline$>=3$ (32 empresas) & $3.46 \mathrm{e}+09$ & 441,71 & 89,25 & 44,03 \\
\hline$<3$ ( 9 empresas) & $3.59 \mathrm{e}+09$ & 467,44 & 136,11 & 52,25 \\
\hline \multicolumn{5}{|c|}{ Criterio 8: Existencia de brecha digital con respecto al resto del sector bancario } \\
\hline$>=3$ ( 23 empresas) & $3.41 \mathrm{e}+09$ & 378,13 & 87,08 & 46,04 \\
\hline$<3$ (18 empresas) & $3.59 e+09$ & 535,83 & 115,44 & 45,37 \\
\hline \multicolumn{5}{|c|}{ Criterio 9: Percepción del impacto de la transformación digital de la economía } \\
\hline >=3 (39 empresas) & $3.62 \mathrm{e}+09$ & 464,35 & 102,51 & 44,38 \\
\hline$<3$ (2 empresas) & $8.83 e+08$ & 116 & 41,50 & 70,50 \\
\hline \multicolumn{5}{|c|}{ Criterio 10: Intensidad de la competencia de los bancos "on line" } \\
\hline >=3 (36 empresas) & $3.82 \mathrm{e}+09$ & 495,41 & 109,83 & 42,48 \\
\hline$<3$ (5 empresas) & $1.09 e+09$ & 101,40 & 25,40 & 67,40 \\
\hline \multicolumn{5}{|c|}{ Criterio 11: Intensidad de la competencia del sector Fintech } \\
\hline$>=3$ (36 empresas) & $3.62 \mathrm{e}+09$ & 459,13 & 99,63 & 44 \\
\hline$<3$ (5 empresas) & $2.52 \mathrm{e}+09$ & 362,60 & 98,80 & 60,75 \\
\hline \multicolumn{5}{|c|}{ Criterio 12: Intensidad de la competencia de las grandes empresas tecnológicas } \\
\hline$>=3$ (33 empresas) & $3.46 \mathrm{e}+09$ & 451,84 & 101,30 & 44,93 \\
\hline$<3$ (8 empresas) & $3.59 \mathrm{e}+09$ & 428,87 & 92,25 & 50,16 \\
\hline \multicolumn{5}{|c|}{ Criterio 13: Grado general de cultura tecnológica } \\
\hline >=3 (20 empresas) & $4.15 \mathrm{e}+09$ & 477,05 & 120,55 & 46,42 \\
\hline$<3$ (21 empresas) & $2.86 \mathrm{e}+09$ & 419,09 & 79,52 & 45,10 \\
\hline \multicolumn{5}{|c|}{ Criterio 14: La entidad puede aumentar la cultura tecnológica de socios y clientes } \\
\hline >=3 (36 empresas) & $2.89 e+09$ & 388,66 & 81,41 & 44,08 \\
\hline$<3$ (5 empresas) & $7.81 e+09$ & 870 & 230 & 60 \\
\hline
\end{tabular}

FUENTE: Elaboración propia a partir de los resultados del cuestionario. 
consideraron un grado alto de asimilación para los componentes vinculados directamente a la entidad (Dirección General y equipo directivo y empleados) cuentan, en términos medios, con un activo total y un número de empleados y oficinas superior al de las organizaciones que asignaron una valoración baja. Sin embargo, la situación se invierte para el activo total si se trata de los componentes sin vínculo directo, como Consejo de Administración y Presidencia y socios y clientes. Por otra parte, también destaca que las empresas que estimaron un grado alto de asimilación de la transformación digital entre los cargos de gestión son, en promedio, más antiguas y presentan mayor dotación de empleados y oficinas que las que indicaron una puntuación baja.

Continuando con la estimación de percepciones de socios y clientes sobre la adaptación tecnológica de la entidad a sus necesidades (criterio 7), las empresas que consideraron una adaptación insuficiente (baja valoración) son, en promedio, mayores en dimensión y más antiguas que las que asignaron una puntuación elevada. Sin embargo, al hablar de brecha digital (criterio 8), son estas empresas con mayor dotación estructural las que perciben menor distancia respecto al resto del sector bancario.

Si se hace referencia a la percepción de la intensidad de la competencia proveniente de empresas cuyo modelo de negocio se sustenta en la tecnología en los próximos cinco años (criterios 9 a 11), destaca que las empresas con mayor número de empleados y oficinas y más jóvenes (en promedio) son las que muestran mayor preocupación otorgando una valoración alta. Por último, respecto al grado de cultura tecnológica de socios y clientes (criterios 13 y 14), las entidades que concedieron una alta valoración presentan un mayor tamaño medio, en términos de activo, empleados y oficinas, que aquellas cuya puntuación asignada fue baja; mientras que las entidades que más confían en contribuir a fomentar dicha cultura presentan, en promedio, menor tamaño y antigüedad que las que manifestaron una puntuación baja respecto a las posibilidades de aumentar la cultura tecnológica de socios y clientes.

Adicionalmente, la tabla 5 complementa la información anterior presentando un análisis de cada uno de los ítems sobre transformación digital, basado en la obtención de los estadísticos media aritmética y percentiles 25,50 y 75 para dos submuestras, que distinguen entre empresas de diferente dimensión: aquellas cuyo número de oficinas y empleados supera a las respectivas medias muestrales y las que, como máximo, alcanzan dicho valor medio. De esta forma, se puede conocer si existen diferencias en las percepciones sobre transformación digital derivadas del tamaño de la entidad. Iniciando este análisis con las percepciones en torno al margen de mejora y la asimilación entre los componentes de la entidad de la transformación digital, en el primer caso se aprecia que las entidades cuyo número de oficinas y empleados es inferior o igual al valor medio muestral valoran de manera muy similar el grado de mejora en la operativa y en los procesos de gestión interna (en el rango 3,53,6 y con percentiles similares). Sin embargo, las cooperativas agrupadas en el conjunto de mayor tamaño consideran que el margen de mejora es más amplio en los procesos de gestión interna, además de que otorgan a este ítem una puntuación superior a la establecida por las entidades que integran el grupo de menor dimensión. En el segundo caso, resulta destacable que las empresas más 


\section{Tabla 5. Análisis del grado de transformación digital según dimensión de la entidad tomando como referencia la media muestral del número de oficinas $(99,53)$ y de empleados $(447,36)$. Estadísticos obtenidos: Media/ Perc25/Perc50/ Perc75}

\begin{tabular}{|c|c|c|c|c|}
\hline \multirow[t]{2}{*}{ Pregunta del cuestionario } & \multicolumn{2}{|c|}{$\mathrm{N}^{\circ}$ Oficinas } & \multicolumn{2}{|c|}{ Empleados } \\
\hline & $<=99,53$ & $>99,53$ & $<=447,36$ & $>447,36$ \\
\hline Margen de mejora en la operativa con productos, servicios y clientes & $3,6 / 3 / 4 / 4$ & $3,36 / 3 / 3 / 4$ & $3,53 / 3 / 4 / 4$ & $3.55 / 3 / 3 / 4$ \\
\hline Margen de mejora en los procesos de gestión interna & $3,6 / 3 / 4 / 4$ & $3,72 / 3 / 4 / 4$ & $3,56 / 3 / 4 / 4$ & $3.88 / 3 / 4 / 5$ \\
\hline $\begin{array}{l}\text { Grado de asimilación de la transformación digital: Consejo de } \\
\text { Administración y Presidencia }\end{array}$ & $2,83 / 2 / 3 / 4$ & $3,09 / 3 / 3 / 3$ & $2,84 / 2 / 3 / 4$ & $3.11 / 3 / 3 / 3$ \\
\hline $\begin{array}{l}\text { Grado de asimilación de la transformación digital: Dirección General } \\
\text { y equipo directivo }\end{array}$ & $3,66 / 3 / 4 / 4$ & $3,54 / 3 / 4 / 4$ & $3,65 / 3 / 4 / 4$ & $3.55 / 3 / 4 / 4$ \\
\hline Grado de asimilación de la transformación digital: Resto de empleados & $3,56 / 3 / 3,5 / 4$ & $3 / 3 / 3 / 3$ & $3,53 / 3 / 3,5 / 4$ & $3 / 3 / 3 / 3$ \\
\hline Grado de asimilación de la transformación digital: Socios y clientes & $2,96 / 2 / 3 / 4$ & $2,36 / 2 / 2 / 3$ & $2,84 / 2 / 3 / 3$ & $2.66 / 2 / 3 / 3$ \\
\hline $\begin{array}{l}\text { Percepción de socios y clientes de adaptación tecnológica a sus } \\
\text { necesidades }\end{array}$ & $3,23 / 3 / 3 / 4$ & $2,63 / 2 / 2 / 4$ & $3,15 / 3 / 3 / 4$ & $2.77 / 2 / 3 / 4$ \\
\hline Existencia de brecha digital con respecto al resto del sector bancario & $2,63 / 2 / 3 / 4$ & $2,63 / 2 / 2 / 4$ & $2,62 / 2 / 3 / 3,5$ & $2.66 / 2 / 2 / 4$ \\
\hline Percepción del impacto de la transformación digital de la economía & $4 / 4 / 4 / 5$ & $4,18 / 4 / 4 / 5$ & $3,96 / 4 / 4 / 5$ & $4.33 / 4 / 4 / 5$ \\
\hline Tecnologías que más afectan a la actividad y su importancia (caso: Big Data) & $4,03 / 4 / 4 / 5$ & $4,18 / 4 / 4 / 5$ & $4,03 / 4 / 4 / 5$ & $4.22 / 4 / 4 / 5$ \\
\hline Tecnologías que más afectan a la actividad y su importancia (caso: Blockchain) & $3,24 / 2 / 3 / 4$ & $3,54 / 3 / 3 / 4$ & $3,25 / 2 / 3 / 4$ & $3.55 / 3 / 3 / 4$ \\
\hline Tecnologías que más afectan a la actividad y su importancia (caso: Criptomonedas) & $2,2 / 1 / 2 / 3$ & $2,45 / 1 / 3 / 3$ & $2,15 / 1 / 2 / 3$ & $2.66 / 2 / 3 / 3$ \\
\hline Tecnologías que más afectan a la actividad y su importancia (caso: Roboadvisor) & $3,2 / 2 / 4 / 4$ & $3,18 / 2 / 3 / 4$ & $3,09 / 2 / 3 / 4$ & $3.55 / 3 / 4 / 4$ \\
\hline Tecnologías que más afectan a la actividad y su importancia (caso: Inteligencia Artificial) & $3,2 / 2 / 3,5 / 4$ & $3,63 / 3 / 3 / 5$ & $3,12 / 2 / 3 / 4$ & $4 / 3 / 4 / 5$ \\
\hline Tecnologías que más afectan a la actividad y su importancia (caso: Internet de las Cosas) & $2,4 / 2 / 2 / 3$ & 2/0/2/4 & $2,28 / 1,5 / 2 / 3$ & $2.33 / 1 / 3 / 4$ \\
\hline Tecnologías que más afectan a la actividad y su importancia (caso: Cloudcomputing) & $3,3 / 3 / 3 / 4$ & $3,45 / 2 / 4 / 5$ & $3,15 / 2,5 / 3 / 4$ & $4 / 4 / 4 / 5$ \\
\hline $\begin{array}{l}\text { Tecnologías que más afectan a la actividad y su importancia } \\
\text { (caso: Realidad aumentada y virtual) }\end{array}$ & $2,26 / 2 / 2 / 3$ & $2 / 1 / 2 / 3$ & $2,18 / 1 / 2 / 3$ & $2.22 / 1 / 2 / 3$ \\
\hline $\begin{array}{l}\text { Tecnologías que más afectan a la actividad y su importancia } \\
\text { (caso: Asistentes virtuales domésticos) }\end{array}$ & $2,46 / 2 / 2 / 3$ & $2,27 / 1 / 2 / 3$ & $2,31 / 1,5 / 2 / 3$ & $2.77 / 2 / 3 / 3$ \\
\hline Intensidad de la competencia desde otras entidades financieras similares & $3,13 / 2 / 3 / 4$ & $3 / 2 / 3 / 4$ & $3,15 / 2 / 3 / 4$ & $2.88 / 2 / 3 / 4$ \\
\hline Intensidad de la competencia de otras entidades bancarias de mayor dimensión & $4,3 / 4 / 4 / 5$ & $4,45 / 4 / 5 / 5$ & $4,37 / 4 / 5 / 5$ & $4.22 / 4 / 4 / 5$ \\
\hline Intensidad de la competencia de la banca extranjera & $2,76 / 2 / 2 / 4$ & $2,18 / 1 / 2 / 3$ & $2,84 / 2 / 2,5 / 4$ & $1.77 / 1 / 2 / 3$ \\
\hline Intensidad de la competencia de los bancos "on line" & $3,86 / 3 / 4 / 5$ & $3,81 / 3 / 4 / 5$ & $3,87 / 3 / 4 / 5$ & $3.77 / 3 / 4 / 4$ \\
\hline Intensidad de la competencia del sector fintech & $3,6 / 3 / 4 / 5$ & $3,72 / 3 / 4 / 5$ & $3,62 / 3 / 4 / 4,5$ & $3.66 / 3 / 4 / 5$ \\
\hline Intensidad de la competencia de las grandes empresas tecnológicas & $3,5 / 3 / 4 / 4$ & $3,81 / 3 / 4 / 5$ & $3,5 / 3 / 4 / 4$ & $3.88 / 4 / 4 / 5$ \\
\hline Grado general de cultura financiera & $2,73 / 2 / 3 / 3$ & $2,90 / 2 / 3 / 4$ & $2,62 / 2 / 3 / 3$ & $3.33 / 3 / 3 / 4$ \\
\hline Grado general de cultura tecnológica & $2,5 / 2 / 2 / 3$ & $2,54 / 2 / 3 / 3$ & $2,5 / 2 / 2 / 3$ & $2.55 / 2 / 3 / 3$ \\
\hline La entidad puede aumentar la cultura financiera de socios y clientes & $3,96 / 4 / 4 / 4$ & $3,36 / 3 / 3 / 4$ & $3,9 / 3,5 / 4 / 4$ & $3.44 / 3 / 3 / 4$ \\
\hline La entidad puede aumentar la cultura tecnológica de socios y clientes & $3,86 / 3 / 4 / 4$ & $3 / 2 / 3 / 4$ & $3,81 / 3 / 4 / 4$ & $2 / 3 / 3 / 3$ \\
\hline
\end{tabular}

FUENTE: Elaboración propia a partir de los resultados del cuestionario.

CIRIEC-España, Revista de Economía Pública, Social y Cooperativa 
grandes perciben un menor grado de asimilación de la transformación digital de los componentes de la organización, a excepción del Consejo de Administración y Presidencia.

A su vez, las empresas del conjunto de mayor tamaño también asignan una peor valoración a la percepción de socios y clientes sobre la adaptación tecnológica de la entidad a sus necesidades de productos y servicios, concretamente, 2,63 frente a 3,23 o 2,77 frente a 3,15; además de percibir un impacto mayor de la transformación digital de la economía. No obstante, si se observan los valores próximos entre las submuestras en torno a la existencia de brecha digital respecto al resto del sector bancario, éstos indican el consenso existente, aunque tal brecha no se considere amplia.

Respecto a las tecnologías que más afectan a la actividad de la entidad financiera, en la mayor parte de los casos, los valores más altos son asignados por el conjunto de empresas de mayor dimensión, es decir, que éstas otorgan más importancia al impacto que las diferentes tecnologías ejercen sobre su actividad, si bien, como se pudo comprobar en el análisis de la tabla 3, no todas se valoran con igual relevancia. Adicionalmente, los ítems relativos a la intensidad de la competencia en los próximos cinco años también arrojan diferencias entre las puntuaciones otorgadas por ambas submuestras. Específicamente, los mayores diferenciales se observan en torno a la competencia proveniente de la banca extranjera, tal que se percibe menos intensa en las entidades de mayor dimensión $(2,18$ frente a 2,76 o 1,77 frente a 2,84). A la vez, la competencia que preocupa más a las entidades más grandes es la proveniente del sector fintech y la de grandes empresas tecnológicas. Por último, las entidades con mayor dotación estructural asignan una mejor valoración al grado de cultura financiera y tecnológica de socios y clientes, aunque se muestran más pesimistas que las del conjunto de menor tamaño al respecto de la capacidad de la entidad de fomentar dicha cultura.

Según lo analizado hasta aquí, se pueden concretar algunos resultados destacables. Por una parte, las cooperativas participantes en este estudio han puesto de manifiesto la necesidad de mejora existente en el sector en cuanto a la transformación digital, así como que los componentes directamente vinculados a la entidad son los que presentan una mayor asimilación de dicha transformación; que existe brecha digital respecto al resto del sector bancario, que son conscientes del impacto significativo de la transformación digital de la economía, que el big data es la tecnología con mayor repercusión en la actividad, que se espera en los próximos cinco años una competencia intensa de las empresas de base tecnológica y que existe una insuficiente cultura tecnológica de socios y clientes. A su vez, las características de las entidades varían según diferentes percepciones sobre la transformación digital. En este sentido, las empresas que afirmaron la existencia de un amplio margen de mejora en el ámbito operativo presentan menor dimensión y más antigüedad que el resto. En el caso de la consideración del alto margen de mejora en los procesos de gestión interna, las respuestas se corresponden con las empresas que, en promedio, poseen mayor activo total y número de empleados.

Adicionalmente, los resultados indican que las percepciones sobre transformación digital aparecen condicionadas por la dimensión de la entidad, ya que las entidades de mayor tamaño estiman mayor necesidad de mejora en los procesos de gestión interna que en la operativa, en general, per- 
ciben un menor grado de asimilación de la transformación digital de los componentes de la organización, le otorgan mayor importancia al impacto de las diferentes tecnologías, perciben menos intensidad de la competencia de la banca extranjera y valoran mejor el grado de cultura tecnológica y financiera de socios y clientes.

Para tratar de complementar al análisis expuesto, se han buscado estudios cualitativos sobre el proceso de transformación digital en el sector bancario, con el fin de realizar una comparativa que permita enriquecer el trabajo mediante la detección de posibles rasgos distintivos en cada ámbito. De esta búsqueda se han detectado muy pocos trabajos, con enfoques diferentes al aquí empleado, lo que hace difícil la comparación. No obstante, del estudio de Herráiz et al. (2015), que se nutre de entrevistas a directivos de grandes bancos españoles, se extraen más bien semejanzas con las respuestas presentadas en este apartado, referidas fundamentalmente a lo mucho que queda por hacer en el sector para lograr la transformación digital y a la importancia del big data para la actividad en este contexto, dada la inmensidad de datos sobre clientes que poseen los bancos y que podrían ser explotados mediante esta tecnología.

En este sentido, se aprecia que el conjunto del sector bancario muestra una clara inquietud con el proceso de transformación digital y percibe los cambios que se están produciendo en el conjunto de la sociedad y, por ende, en sus clientes y usuarios. La tradicional competencia intra-sectorial entre entidades bancarias y financieras comienza a ampliarse de modo inter-sectorial, involucrando a nuevos actores en los que el componente tecnológico es el elemento diferenciador; tanto desde el lado de las empresas fintech (generalmente nuevas y pequeñas entidades financieras basadas en aplicaciones tecnológicas) como desde el lado de las empresas puramente tecnológicas que comienzan a operar en el ámbito financiero (como es el caso de las grandes bigtech que originan el apelativo de techfin).

En este escenario, identificado por el acrónimo "VUCA", acuñado en los tiempos de la la guerra fría (Volatility-Uncertainty-Complexity-Ambiguity), la banca en general y consiguientemente, la banca cooperativa, se ven obligados a redefinir su estrategia comercial e, incluso, parte de su modelo de negocio.

Las cooperativas de crédito, habitualmente con menor dimensión comparativa con respecto a la gran banca comercial y basadas en modelos federativos, de cooperación o de sistemas de concentración cooperativa en los que las entidades y sus usuarios otorgan un valor especial a la autoidentificación territorial y la cercanía geográfica y relacional con el socio, la tecnología puede ser un factor de distanciamiento y de pérdida de contacto personal con el socio o cliente. Por ello, dado que el avance tecnológico no puede ser frenado y carece de sentido negarlo o intentar evitarlo, la banca cooperativa debe diseñar estrategias de compatibilidad entre la filosofía de su particular modelo de negocio y la asimilación de la transformación digital. Precisamente, su cercanía al cliente puede ser el modo de adquirir un papel destacado en el proceso de educación financiero-digital que la sociedad va a requerir y que actualmente preocupa incluso a las instituciones supervisoras ${ }^{8}$, pudiendo desempeñar un nuevo rol de cercanía basado en su papel de catalizador y difusor de la digitalización de la economía. 
Las cooperativas de crédito deben valorar diversas líneas de transformación digital de su actividad, tanto en sus procesos internos y organizativos como en sus procesos de gestión con clientes. En este último caso, la reducción de plazos de gestión, la utilización de $A p p s^{9}$ y de sistemas de acceso cada vez más sencillos, ágiles, cómodos y de uso atractivo resulta esencial. Se debe atender, muy especialmente, a todo aquello que implique una experiencia de usuario más satisfactoria y la simplificación de los trámites y gestiones, con una oferta cada vez más personalizada.

En la definición de las estrategias de transformación digital se debe establecer un horizonte adecuado con desarrollos tácticos específicos, pero con un plan a medio y largo plazo consistente. Sin duda alguna, las cooperativas de crédito tendrán que desarrollar internamente y con el apoyo de empresas especializadas un plan de transformación digital que vaya más allá de la digitalización de algunos procesos. Deberán mirar hacia dónde se dirigen y dónde se va a aportar valor con esa transformación. En este sentido, la estrategia pasa por comenzar a valorar nuevas tecnologías que ya anticipan grandes cambios, como es el caso de blockchain en sus procesos internos; pero también comenzar a utilizar la ingente cantidad de datos que recogen de la relación con los clientes al objeto de mejorar la personalización de la oferta (aplicación de big data y Smart data).

Otro elemento disruptor a valorar en su estrategia es el desarrollo del modelo de supermercado financiero, ahora cada vez más viable con las nuevas tecnologías. Los sistemas de identidad digital criptográficas de las DLT y blockchain (o cadenas de bloques) van a simplificar en pocos años los procesos de on-boarding (el registro o proceso de alta de un nuevo usuario) y de KYC (Know Your Costumer 0 análisis de cliente); lo que permitirá la contratación de productos y servicios financieros en diversas entidades sin que sea preciso más que una única identificación en una de ellas y, probablemente, sin que se requiera el tradicional paso previo de registro como cliente. Esto va a intensificar la competencia y convertirá los servicios financieros en una mera "utility" fácilmente desintermediable y con capacidad de ser ofrecida por competidores financieros y tecnológicos. del Mercado de Valores, similar al que realizan sus homólogos en otros países europeos (Comisión Nacional del Mercado de Valores, 2018).

9. - Abreviatura de application en inglés, referido a aplicaciones para dispositivos móviles. 


\section{Conclusiones}

Este trabajo constituye un análisis del impacto de la transformación digital en el sector financiero, centrado en un segmento de banca minorista tradicional como es la banca cooperativa.

Las cooperativas de crédito fundamentan su modelo de intermediación financiera en la proximidad física a sus socios y clientes mediante una red física de oficinas que cubre los ámbitos territoriales en los que han nacido y se han desarrollado, algunas de ellas, durante más de 100 años. Con la excepción de algunas entidades, la mayoría de ellas operan en ámbitos provinciales o autonómicos, aunque abundan también las que tienen ámbitos de actuación más limitados de carácter local o comarcal.

Por esta razón es relevante analizar cómo la transformación digital, la banca a distancia y el tratamiento automatizado de muchas operaciones financieras, que ya no requieren la presencia física de los usuarios en las oficinas, están repercutiendo o pueden afectar al desarrollo futuro de su actividad y a su estrategia competitiva. Además, hay que tener en cuenta que, si hasta hace pocos años, la competencia procedía de otras redes comerciales de oficinas bancarias, ahora la presión de la competencia puede venir de una empresa fintech o de los servicios financieros que pueda desarrollar cualquier gran compañía tecnológica.

Del análisis realizado mediante la realización de un cuestionario y la obtención de estadísticos descriptivos se desprende que, el conjunto de las cooperativas de crédito españolas analizadas reconocen que deben afrontar el reto de la transformación tecnológica de la economía y de la sociedad, si bien, entienden que el ritmo de asimilación de este desarrollo tecnológico en su actividad es, en algunos casos, superior al de sus socios y clientes.

A efectos competitivos, consideran que tienen una leve brecha digital con respecto al resto del sector bancario y que son conscientes del impacto que determinados desarrollos tecnológicos van a tener en su modelo de negocio. En particular destacan, en este orden: bigdata, computación en la nube y blockchain.

A su vez, son conscientes de que el nuevo escenario competitivo ha cambiado sustancialmente, pues no sólo el resto de bancos son competidores directos, sino que también emergen en ese escenario nuevos actores hasta hace poco impensables e inesperados: las grandes compañías tecnológicas, que ahora comienzan a ofrecer servicios financieros además de su enorme capacidad de computación y aprovechamiento de los datos de sus millones de usuarios; así como competidores de minúsculo tamaño comparativo, pero con el poder de aplicar algoritmos y robotización mediante 
sistemas de ínfimo coste laboral comparativo, así como el acierto en la prestación de servicios que el usuario valora muy positivamente porque percibe una muy satisfactoria experiencia de uso con un menor coste por la intermediación.

Por otra parte, es interesante apreciar que hay diferencias de opinión relativas con respecto a determinados aspectos de la transformación digital cuando varía la dimensión de las entidades encuestadas. Específicamente, las cooperativas de crédito más pequeñas parecen ser menos sensibles a la necesidad de mejora en los procesos de gestión interna, lo cual puede indicar la necesidad de buscar mayores sinergias que una reducida dimensión puede impedir; y conseguir una adecuada escalabilidad en sus procesos. La dimensión también influye en la percepción sobre la asimilación de la transformación digital entre los componentes de la entidad, estando mejor valorada, en general, por las cooperativas de menor tamaño. También las más grandes están más preocupadas por la irrupción de las fintech y de las grandes tecnológicas.

En suma, las cooperativas de crédito españolas son conscientes de los importantes cambios que van a acontecer en un futuro casi inmediato como consecuencia de la acelerada evolución de las tecnologías aplicables en el ámbito financiero. Asumen también que su modelo de intermediación no sólo sufrirá la competencia de las restantes entidades bancarias de su sector de actividad -como ocurría hasta ahora- sino que también provendrá de las nuevas empresas del sector fintech y de agentes externos no financieros pero con un enorme poder, como son las grandes compañías tecnológicas, que han descubierto un nicho de mercado importante en el ámbito financiero y sobre el que pueden volcar muchas de las herramientas que han desarrollado en los últimos años, teniendo a los datos como principal activo.

El modelo de banca tradicional basado en la relación personal directa entre los empleados y los socios y clientes, fundamentado en la red de oficinas, parece amenazado en algunas parcelas por la suplantación que ejercen los sistemas de banca a distancia, los algoritmos de procesamiento automatizado y la inteligencia artificial; lo cual obligará a la banca cooperativa a reforzar su identidad y su forma de interactuar con sus socios y clientes.

Para finalizar, conviene reseñar las limitaciones que han condicionado esta investigación, las cuales se concretan, fundamentalmente, en que el análisis se basa en percepciones de los directivos de las entidades encuestadas, lo cual implica un grado de subjetividad condicionada por múltiples variables no controlables; $y$ en que se trata de una aproximación general a diferentes aspectos relacionados con la transformación digital, por lo que se no se han podido contemplar otras cuestiones trascendentes. Como líneas futuras se contempla un análisis más amplio tomando muestras de usuarios de socios y clientes de estas entidades, así como una investigación sobre un período temporal determinado que valore la evolución de la transformación digital, los cambios en la demanda de los usuarios de servicios bancarios y, también, cuáles son las tecnologías que más inciden o que se van a utilizar en el desarrollo de la actividad de las cooperativas de crédito. 


\section{Anexo 1. Ficha técnica de la muestra.}

Universo

Cooperativas de crédito españolas

Tamaño de la población 62 entidades

Tamaño de la muestra .41 entidades

Metodología .Encuesta online anónima (plataforma Eval\&Go)

Período de realización .20/03/2018-25/04/2018

Preguntas .20

FUENTE: Elaboración propia.

\section{Bibliografía}

AKKIZIDIS, I. \& STAGARS, M. (2016): Marketplace lending, Financial Analysis, and the Future of credit: Integration, Profitability, and risk management, John Wiley Sons Inc, Estados Unidos.

BANSAL, N. \& JAIN, M. (2018): "Progress in Digital Banking After Demonetization: Some Evidence", IUP Journal of Bank Management, 17(2), 50-59.

BBVA RESEARCH (2017): De Alan Turing al ciberpunk: la historia de Blockchain. Recuperado de: https://www.bbva.com/es/historia-origen-blockchain-bitcoin/, 15 diciembre de 2017.

BLOMSTROM, D. (2018): Emotional banking: Fixing culture, leveraging FinTech, and transforming retail banks into brands, Palgrave Macmillan, London. DOI:10.1007/978-3-319-75653-0.

BUSBY, D. (2017): "Adopting the best approach for a digital banking solution: Combine the benefits of the 'build', 'buy'or 'outsource'options", Journal of Digital Banking, 2(1), 43-50.

CARBÓ, S. \& RODRÍGUEZ-FERNÁNDEZ, F. (2017): "La banca española en Europa: de la transición a la acción", Cuadernos de Información Económica, 257,1-9.

CARBÓ, S. \& RODRÍGUEZ-FERNÁNDEZ, F. (2014): "El sector bancario español ante un nuevo paradigma: Reconsideración del tamaño", Papeles de Economía Española, Número extraordinario: Nuevos negocios bancarios, 19-20.

CARBÓ, S. \& RODRÍGUEZ-FERNÁNDEZ, F. (2016): "Otro giro en la reestructuración bancaria", Cuadernos de Información Económica, 252, 84-97. 
CHAVES, R. \& SOLER, F. (2004): El gobierno de las cooperativas de crédito en España, CIRIECEspaña, Valencia.

CHESBROUGH, H. (2003): Open Innovation: The new imperative for creating and profiting from technologies, Harvard Business School Press, Cambridge-MA.

CHESBROUGH, H. (2010): "Business model innovation, opportunities and barriers", Long Range Planning, 43, 354-363. https://doi.org/10.1016/j.lrp.2009.07.010.

CHISHTI, S. \& BARBERIS, J. (2016): The Fintech book, John Wiley \& Sons, West Sussex.

CHRISTENSEN, M., RAYNOR, M. \& McDONALD, R. (2015): "What is Disruptive Innovation", Harvard Business Review, 93(12), 44-53.

COMISIÓN NACIONAL DEL MERCADO DE VALORES (2018): Plan de Educación Financiera de la CNMV y el Banco de España, Recuperado de: https://www.cnmv.es/portal/Publicaciones/PlanEducCNMV.aspx, 2 de marzo de 2018.

DAPP, T.M. (2015): Fintech reloaded - Traditional banks as digital ecosystems: With proven walled garden strategies into the future. Recuperado de:

https://www.deutschebank.n//n//docs/Fintech_reloaded_Traditional_banks_as_digital_ecosystems.pdf, 27 de mayo de 2018.

EACB-EUROPEAN ASOCIATION OF COOPERATIVE BANKS (2014): Road Map for Co-operative Banks, EACB, Bruselas.

EVANS, PH. \& WURSTER, TH.S. (1999): Blown to Bits, Harvard Business School Press, Boston.

FAJARDO, G. \& SOLER, F. (2016): "The Credit Cooperative System in Spain". In: KARAFOLAS, S. (Ed.), Credit Cooperative Institutions in European Countries, 213-232, Springer.

GIESEN, E., RIDDLEBERGER, E., CHRISTENSEN, R. \& BELL, R. (2010): "When and how to innovate your business model", Strategy and Leadership, 38(4), 17-26.

https://doi.org/10.1108/10878571011059700.

GOMBER, P., KAUFFMAN, R.J., PARKER, C. \& WEBER, B.W. (2018): "On the Fintech Revolution: Interpreting the Forces of Innovation, Disruption, and Transformation in Financial Services", Journal of Management Information Systems, 35(1), 220-265.

GUTIÉRREZ, M. \& PALOMO, R. (2012): "Los sistemas institucionales de protección como respuesta de la banca cooperativa española ante la crisis: hacia la búsqueda de la eficiencia en su integración", CIRIEC-España, Revista de Economía, Pública, Social y Cooperativa, 76, 27-50.

GUTIÉRREZ, M., PALOMO, R. \& ROMERO, M. (2012): "La expansión territorial como factor motivador de la reestructuración del sistema financiero español: El caso de las cajas de ahorros y las cooperativas de crédito", REVESCO, Revista de Estudios Cooperativos, 107, 7-34. 10.5209/rev_REVE.2012.v107.38746. 
HERRÁIZ, A., GARCÍA, R., CASTEJÓN, L., RODRÍGUEZ, E., ÁLVAREZ, R., OCHOA, B., DE SALAS, A., ROBREDO, M., GARCÍA, A., BOLÍVAR, J., ACEITUNO, L., SEBASTIÁN, J., TSOUROULAS, N., ALARCÓN, P., DEL ÁRBOL, L.P., BERMEJO, E., DELGADO, J., BUENO, F., RIVERA, P., DORTA, P. \& VICARIO, J.L. (2015): La transformación digital de la banca española, Estudio Banca Digital España 2015, Madrid.

HM TREASURY (2015): Digital Currencies: Response to the call for information, HM TREASURY, Reino Unido.

JAKSIC, M. \& MANRIC, M. (2018). "Relationship banking and information technology: The role of artificial intelligence and FinTech", Risk Management, 1-18. DOI:10.1057/s41283-018-0039-y

JAUBERT, M., MARCUS, S., ULLRICH, M. \& MALBATE, J.B. (2014): Going Digital: The Banking Transformation Roadmap. Recuperado de: https://www.atkearney.com/documents/10192/5264096/ Going+Digital+-+The+Banking+Transformation+Road+Map.pdf/60705e64-94bc-44e8-9417652ab318b233, 10 Junio de 2018.

KING, B. (2014): Breaking Banks: The innovators, Rogues, and strategists rebooting banking, John Wiley Sons Inc, Estados Unidos.

LERIDA, J. \& MORA, J.J. (2016): La economía de blockchain: los modelos de negocios de la nueva web, Kolokium, Madrid.

MASSANELL, J.A. (2016): "La transformación de la banca: reorientación de los canales y servicios digitales", Papeles de Economía Española, 149, 93-99.

MAUDOS, J. (2017): "Modelos de negocio en la banca europea: cambios", Cuadernos de Información Económica, 257, 11-25.

MAY, T.C. (1992): The Crypto Anarchist Manifesto. Recuperado de: http://nakamotoinstitute.org/literature/crypto-anarchist-manifesto/, 25 de junio de 2018.

MBAMA, C.I. \& EZEPUE, P.O. (2018): "Digital banking, customer experience and bank financial performance: UK customers' perceptions", International Journal of Bank Marketing, 36(2), 230-255. https://doi.org/10.1108//JBM-11-2016-0181.

MORRIS, L. (2006): "Business Model Innovation: The Strategy of Business Breakthroughs", International Journal of Innovation Science, 1(4), 191-204. https://doi.org/10.1260/1757-2223.1.4.191.

MUKHERJEE, A.\& NATH, P. (2003): "A model of trust in online relationship banking", International journal of bank marketing, 21(1), 5-15. https://doi.org/10.1108/02652320310457767.

OLANREWAJU, T. (2014): "The rise of the digital bank", McKinsey on Business Technology, 33. Recuperado de: https://www.mckinsey.com/business-functions/digital-mckinsey/our-insights/therise-of-the-digital-bank, 15 junio de 2018. 
OLIVER WYMAN (2014): Cooperative Banking: Leveraging the cooperative difference to adapt to a new environment, Oliver Wyman Financial Services.

PALOMO, R. (2017): "El cooperativismo de crédito frente a los retos del sector financiero: desafíos y oportunidades en un nuevo entorno socio-tecnológico". In: Maudos, J. (Ed.), El futuro del sector bancario español tras la reestructuración, 289-310, Cajamar Caja Rural.

PALOMO, R.J. \& SANCHIS, J.R. (2010): "Efectos de las fusiones sobre la concentración y la eficiencia bancaria: El caso de las cajas rurales y los retos de la crisis financiera", Revista Española de Financiación y Contabilidad, 146, 291-321.

RAHMAN, A.M. (2017): "Incentive under market system along with informational symmetry to boost up customer attitude toward self-service banking: a proposal", International Journal of Economics and Business Research,14(3-4), 312-331. https://doi.org/10.1504/JJEBR.2017.087518.

SANCHIS, J.R. (2016): ¿Es posible un mundo sin bancos?, El Viejo Topo, Valencia.

SHAIKH, A.A., GLAVEE-GEO, R. \& KARJALUOTO, H. (2017): "Exploring the nexus between financial sector reforms and the emergence of digital banking culture-Evidences from a developing country", Research in International Business and Finance, 42, 1030-1039. https://doi.org/10.1016/j.ribaf.2017.07.039.

SKINNE, C. (2016): How FINTECH firms are using Mobile and Blochchain Technologies to create the internet of value, Marshall Cavendish International, Singapore.

TAYLOR, K.C. (2014): Fin Tech Law: A guide to technology law in the financial services industry, Bloomberg Bna, Estados Unidos.

THE ECONOMIST LEADERS (2015): The Fintech Revolution. Recuperado de: https://www.economist.com/leaders/2015/05/09/the-fintech-revolution, 26 de junio de 2018.

UNACC (2000-2017): Anuario estadístico de las Cooperativas de Crédito, Unión Nacional de Cooperativas de Crédito. Recuperado de: http://www.unacc.com/inicio/Publicaciones/Anuario.aspx., 25 de mayo de 2018.

ZACHARIADIS, M. \& OZCAN, P. (2017): "The API Economy and Digital Transformation in Financial Services: The Case of Open Banking", SWIFT Institute Working Paper, 2016-001. http://dx.doi.org/10.2139/ssrn.2975199.

ZOTT, C. \& AMIT, R. (2007): "Business model design and the performance of intrepreneurial firms", Organization Science, 18, 181-199, https://doi.org/10.1287/orsc.1060.0232. 